\title{
Desigualdades sociales y trayectorias de rendimiento académico en niños y niñas de la ciudad de Córdoba, Argentina
}

\author{
EMILSE DEGOY(1) \\ SILVINA BERRA ${ }^{(2)}$
}

Fecha de recepción: 14/02/2020

Fecha de aceptación: 25/08/2020

Resumen. En un contexto de pobreza pero de alta tasa de escolarización, se examina si existen diferentes trayectorias de rendimiento académico (RA) asociadas a características socioeconómicas en niños/as de Córdoba-Argentina. Se realizó un estudio de cohortes prospectivas con escolares, en el que se obtuvieron mediciones anuales del rendimiento académico y mediciones basales de características sociales: sexo, escolaridad materna, estrato ocupacional, nivel socioeconómico (NSE) y capacidad intelectual (CI). Se definieron cuatro trayectorias de RA a partir de las calificaciones en Lengua y Matemática obtenidas por los/as niños/as al inicio y final del seguimiento, y su condición de Promovidos/ No promovidos en cada ciclo lectivo: distinguida, satis-

(1) Licenciada en Psicología por la Facultad de Psicología de la Universidad Nacional de Córdoba (UNC). Doctora en Ciencias de la Salud por Facultad de Ciencias Médicas (FCM) de la UNC. Investigadora en el Centro de Investigación Epidemiológica y en Servicios de Salud de la Escuela de Salud Pública, FCM, UNC. Ha sido Becaria Doctoral del Consejo Nacional de Investigaciones Científicas y Técnicas (CONICET), y Profesional Adscripta en la Cátedra de Metodología de la Investigación, Facultad de Psicología, UNC.

edegoy@gmail.com factoria, regular, insatisfactoria. Se utilizaron modelos de regresión logística binomial y multinomial para examinar la presencia de desigualdades sociales. Se encontraron diferencias significativas en la trayectoria de RA según las variables socioeconómicas estudiadas cuyo efecto no fue independiente de la capacidad intelectual. A su vez, la capacidad intelectual se vio fuertemente asociada con la escolaridad materna, estrato ocupacional y NSE. Se corrobora la presencia de desigualdades sociales en la $\mathrm{Cl}$ y las trayectorias de rendimiento académico de los escolares.

Palabras clave. desigualdades sociales - rendimiento académico $\cdot$ trayectorias $\cdot$ infancia $\cdot$ escuela primaria

(2) Licenciada en Nutrición, Máster en Salud Pública, Doctora en Ciencias de la Salud. Profesora Titular (DS) de Metodología de la Investigación, Escuela de Fonoaudiología; Profesora Adjunta (DS) en la Escuela de Salud Pública de la Facultad de Ciencias Médicas (FCM) en la Universidad Nacional de Córdoba (UNC). Investigadora Adjunta de la Carrera de Investigador Científico del Consejo Nacional de Investigaciones Científicas y Técnicas (CONICET) en el Centro de Investigaciones y Estudios sobre Cultura y Sociedad (CIECS).

sberra@unc.edu.ar 


\section{Social Inequality and Academic Achievement Trajectories of Schoolchildren in the City of Córdoba, Argentina}

\begin{abstract}
In a context of poverty but with a high schooling rate, this work is intended to elucidate if children in Córdoba, Argentina, show differences in their academic achievement (AA) trajectories associated with socioeconomic characteristics. A prospective cohort study was conducted with schoolchildren, in which annual measurements of $A A$ and baseline measurements of social characteristics were collected: sex, mother's educational level, parental occupational stratum, socioeconomic level (SEL) and intellectual capacity (IC). Four categories were defined for academic achievement trajectories according to their scores obtained in the subjects of Language and Mathematics at the beginning and end of the conducted analysis, and in relation to their promotion or not to the next grade by the end of each school year. The four categories for trajectories were: distinguished,
\end{abstract}

satisfactory, regular, and unsatisfactory. Binomial and multinomial logistic regression models were used to examine the presence of social inequalities. Significant differences were found in academic achievement trajectories depending on the studied socioeconomic variables, whose effects were not independent of the intellectual capacity. Intellectual capacity was strongly associated with mother's educational level, parental occupational stratum, and SEL. The influence of social inequalities on intellectual capacity and academic achievement trajectory was proved.

Keywords. social inequalities - academic performance $\cdot$ trajectories $\cdot$ childhood . primary school

\section{Introducción}

La investigación sobre el desarrollo humano ha tenido en la epidemiología del ciclo de la vida un aporte teórico-metodológico para lograr visiones más dinámicas de la salud y contemplar diferentes experiencias en la vida temprana conjuntamente con los de la vida posterior para identificar procesos de riesgo y protección que se van sucediendo a lo largo de la vida (Kuh et al., 2003). En ese marco, las interrelaciones entre educación y salud son descritas mediante diferentes mecanismos (Suhrcke y De Paz Nieves, 2011; Lynch y von Hippel, 2016) y, a su vez, se les reconoce un impacto en las diferencias que puedan encontrarse en la vida adulta. Incluso se señala que la experiencia escolar puede expresar desigualdades sociales en salud, en tanto posibilidades distintas de asistir a la escuela, diferentes niveles de preparación para aprender y dispares resultados escolares (Rajmil et 
al. 2010). En el marco de un proyecto de salud infantil, en el ámbito de escuelas primarias públicas municipales de la ciudad de Córdoba, Argentina (Degoy y Berra, 2018), este trabajo emerge por la inquietud de conocer el efecto que pueden tener determinantes sociales en un trayecto educativo, como parte de una tesis doctoral ${ }^{(3)}$.

La educación primaria en Argentina se ha destacado históricamente por su rápida y amplia expansión. Sin embargo, para garantizar el derecho a la educación, el acceso a la escuela debe complementarse con la generación de condiciones adecuadas para asegurar la continuidad y calidad en las trayectorias educativas de la población. En este sentido, es necesario distinguir entre dos tipos principales de exclusión educativa: las que expresan inequidades de acceso y permanencia, y las que expresan inequidades de progresión. Esta última, hace referencia a la exclusión latente, potencial o silenciosa de la educación primaria y secundaria e incluye a aquellos niños, niñas y adolescentes que se mantienen escolarizados pero con una experiencia educativa signada por fracasos y, como consecuencia de ello, en riesgo educativo (UNESCO-UNICEF, 2012).

El reconocimiento de estos tipos de exclusión es fundamental en los países que han alcanzado o están próximos a alcanzar la escolarización universal de sus niños, niñas y adolescentes ya que, en estos casos, el interrogante sobre la (in)equidad educativa se desplaza casi totalmente hacia el perfil de distribución de los logros de aprendizajes curriculares (Cervini, 2002). En Argentina, en el año 2016, el 98,7\% de los niños y niñas entre 6 y 11 años de edad asistía a la escuela primaria, y no había diferencias en la tasa de escolarización según su pertenencia a los distintos estratos socio-económicos. Sin embargo, las tendencias en los ritmos y la calidad de los aprendizajes obtenidos por los más chicos son preocupantes, según lo revelan informes con resultados de la Evaluación Nacional Aprender (Ministerio de Educación, 2016; en cumplimiento con la Ley 26.206) al señalar un aumento de la tasa de repitencia en el nivel primario de educación para el conjunto del país, y resultados poco satisfactorios de los escolares en materias troncales como Lengua y Matemática, siendo más frecuente este tipo de resultados entre los/as niños/as de sectores más carenciados (Cardini, Sanchez y Rivas, 2016).
(3) Trayectorias de rendimiento académico y salud autopercibida en niños y niñas asistentes a escuelas municipales de la ciudad de Córdoba (Argentina). Un estudio de dos años de seguimiento. Tesis de Doctorado en Ciencias de la Salud, Facultad de Ciencias Médicas, Universidad Nacional de Córdoba (Argentina). Dirigida por la Dra. Silvina Berra. Defendida el 12 de Agosto de 2019. 
En este escenario, avanzar en la construcción de la equidad educativa requiere hacer foco en las desigualdades sociales que puedan producirse una vez dentro de la escuela, esto es, en los logros y trayectorias escolares diferenciales que puedan surgir a partir de diferencias socio-económico-culturales propias de los/as alumnos/as (López, 2005), pero que pueden colocar a ciertos grupos de niños y niñas en una situación de desventaja educativa. El rendimiento académico (RA), desde una perspectiva clásica, es definido como un indicador del aprendizaje alcanzado por el alumno y es el criterio que tradicionalmente se ha adoptado para medir el éxito/fracaso escolar (Navarro, 2003). Con ello, entendemos que, tal y como se encuentra estructurado nuestro sistema educativo, el RA constituye su núcleo duro y es lo que habilita el progreso y la continuidad dentro de él. El bajo desempeño académico y su corolario, el fracaso escolar, refieren a alumnos que no logran el rendimiento deseado dentro del tiempo estipulado $y$, consecuentemente, aparecen como malos estudiantes (Gonzalez-Pienda, 2003). Algunas veces se trata de una situación poco duradera y transitoria; otras veces, es recurrente y sus resultados negativos pueden comprometer sus estudios y porvenir (Terigi, 2009).

En este estudio, se utiliza el término trayectoria de rendimiento académico para referir al despliegue temporal de las calificaciones obtenidas por los niños y niñas. De acuerdo con Briscioli (2017), la idea de trayectoria es un concepto teórico metodológico amplio que, más allá de la temática particular abordada, centra su atención en la interpretación de los fenómenos a lo largo del tiempo, busca comprenderlos en su multidimensionalidad y tiene en cuenta el entramado existente entre las distintas esferas de la vida de las personas.

En la actualidad, se reconoce que el desempeño escolar es un fenómeno complejo y multideterminado por factores tanto de tipo personal como contextual. Dentro de los factores contextuales se suele distinguir entre variables socio-ambientales, institucionales e instruccionales. Las variables socio-ambientales refieren al estatus social, familiar y económico que se dan en el medio lingüístico y cultural específico en el que se desarrolla el individuo. Algunos autores sostienen que, dentro de estas, la familia es la que mayor peso tiene en relación al rendimiento escolar, ya que en su seno es donde se 
inicia el proceso de socialización, se aprenden los primeros roles, normas y modelos de conducta, se empieza a conformar la imagen de sí mismo y la jerarquía de valores que cada niño y niña pondrá en práctica a la hora de su aprendizaje y desempeño dentro de la escuela (Gonzalez-Pienda, 2003). Mientras una gran parte de los trabajos interesados en indagar el modo en que la familia influye tanto en el desarrollo como en los resultados escolares de los/as niño/as han puesto foco en las características psicosociales del ambiente familiar (por ejemplo, características de la relación entre padres e hijos o el nivel de implicación de los padres en la educación de sus hijos), otros han hecho énfasis en sus características estructurales como la posición social, nivel cultural, recursos económicos, etcétera (Gonzalez-Pienda, 2003). En este sentido, estudios realizados en otros contextos y con énfasis en las características estructurales del ambiente familiar señalan una fuerte asociación entre variables socioeconómicas y el desarrollo de las habilidades cognitivas necesarias para superar las exigencias de la escuela (Noble, Norman y Farah, 2005), mientras que otros reconocen al nivel socioeconómico y la escolaridad materna como importantes predictores del éxito escolar de los/as niños/as (Greg, Morris y Rodrigues, 2011; Magnuson, 2007; DavisKean, 2005).

En Argentina, sin embargo, hasta donde se conoce, es poco claro el efecto atribuible al nivel de pobreza/riqueza relativa del hogar sobre los resultados del aprendizaje (Gertel, 2006). Aún se observa una primacía de estudios y modelos explicativos de rendimiento escolar centrados en las características individuales del alumno, siendo pocos los trabajos que han indagado en qué medida variables socioeconómicas influyen en los logros escolares de los más jóvenes (Krüger, 2013) y sin incorporar a las habilidades cognitivas en el análisis de esta relación. Si bien la asociación entre rendimiento escolar y aptitudes cognitivas, en particular la capacidad intelectual, ha sido ampliamente constatada (Lemos et al., 2008), la incorporación de lo contextual lleva a pensar que, mientras la capacidad intelectual afecta el rendimiento académico, los aspectos socio-económico-culturales condicionan ambos, al desempeño escolar y la capacidad intelectual de niños y niñas.

Por lo anteriormente expuesto, este trabajo plantea como objetivo examinar la presencia de desigualdades sociales en la capacidad intelectual y las tra- 
yectorias de rendimiento académico durante el Segundo Ciclo de Escolaridad Primaria (4to a 6to grado) en niños y niñas asistentes a escuelas públicas municipales de la ciudad de Córdoba-Argentina.

\section{Método}

\section{Diseño y participantes}

Se realizó un estudio de cohortes prospectivas en el ámbito de escuelas primarias públicas municipales de la ciudad de Córdoba-Argentina, que inició en el año 2014 cuando los participantes asistían a 4to grado, con seguimiento hasta el 2016, durante el cual se obtuvieron mediciones anuales de la variable rendimiento académico, y mediciones basales de las características sociales.

El sistema educativo municipal cuenta con un total de 37 escuelas, cuya población proviene de familias en situación de mayor desventaja socioeconómica respecto del resto de escuelas de la ciudad (públicas provinciales o de gestión privada), y la cual reside mayoritariamente en barrios de la periferia urbana. La población del estudio fueron alumnos regulares de $4^{\circ}$ grado de escuelas públicas municipales de la ciudad de Córdoba que, en 2014, contaban con una matrícula de 1594 niños/as. Para el proceso de muestreo, en estudios anteriores se estratificaron las escuelas existentes en dos grupos: aquellas con más del $75 \%$ de padres con al menos educación primaria completa $(n=20)$ y las que están por debajo de este umbral $(n=17)$. Diez escuelas fueron seleccionadas al azar del primer grupo y nueve del segundo. De estas 19 escuelas, se eligieron las 10 con mayor matrícula para realizar este estudio. De este modo, la muestra incluyó a todos los estudiantes de $4^{\circ}$ grado de las escuelas seleccionadas, alcanzando un grupo de 533 escolares. 


\section{Procedimiento}

Los objetivos y las características del estudio se explicaron a directivos de las escuelas y cuidadores del niño (madre, padre o tutor legal) durante una reunión escolar. Cuidadores que no asistieron a la reunión recibieron una nota explicando el estudio, invitándolos a participar y proporcionándoles distintos medios de contacto con las investigadoras responsables en caso de dudas e inquietudes. Después de suministrar toda la información necesaria sobre el estudio a la comunidad escolar, se gestionó un consentimiento informado comunitario.

La recolección de datos se realizó en las escuelas e implicó dos instancias. La primera, incluyó una primer visita de presentación de las investigadoras y del estudio a los escolares, en donde además, se les entregó una encuesta en sobre cerrado para sus cuidadores solicitándoles que se la hagan llegar por su intermedio, y una vez completa, la traigan de vuelta, también en sobre cerrado, a la escuela; una segunda visita, en la cual personal técnico entrenado recogió las encuestas de adultos completas y llevó a cabo una administración colectiva guiada en el aula de cuestionarios para niños y niñas; y una tercer/cuarta visita para recuperar cuestionarios de adultos completos faltantes y administrar cuestionarios a niños/as ausentes en la visita anterior. La segunda instancia de recolección de datos consistió en, una vez finalizado cada ciclo lectivo comprendido en el seguimiento, solicitar a las escuelas el registro de calificaciones finales de los/as niños/ as del año lectivo anterior.

Durante todas las etapas de la investigación, se respetaron las Directrices para la Investigación en Salud Humana del Ministerio de Salud de Argentina, y el protocolo de trabajo fue aprobado por el Comité de Ética para la Investigación en Salud de Niños y Adultos (CIEIS del niño y del adulto - Polo Hospitalario) en abril de 2012.

\section{Variables e instrumentos}

Rendimiento académico (RA): se determinó a partir de las calificaciones alcanzadas por los/as niños/as al concluir el año escolar en las materias de Lengua escrita y Matemática aritmética, las cuales se obtuvieron de los registros escolares proporcionados por las oficinas administrativas de las 
escuelas participantes del estudio. En el nivel primario de educación de la Provincia de Córdoba, los/as niños/as son evaluados por sus docentes usando una escala cualitativa ordinal con 5 niveles: No Satisfactorio (NS), Satisfactorio (S), Bueno (B), Muy Bueno (MB), y Excelente (E), debiendo aprobar todas las materias de la currícula con al menos una calificación de Satisfactorio para pasar al siguiente grado. Si en el último trimestre del ciclo lectivo, el/la niño/a obtiene un No Satisfactorio en alguna de las asignaturas de la currícula, debe rendir un examen complementario; si lo aprueba es promovido al grado siguiente, y si lo desaprueba debe re-cursar el grado, o como lo que frecuentemente se denomina repetir de grado.

En este trabajo se seleccionaron las asignaturas de Lengua y Matemática porque constituyen núcleos de aprendizaje prioritarios (Ministerio de Educación, Ciencia y Tecnología, 2005), y porque son las materias en las que los escolares presentan con mayor frecuencia calificaciones No Satisfactorias, convirtiéndose en las asignaturas decisivas para la promoción de un grado a otro, y por ende, para el progreso dentro del nivel primario de educación. En el momento basal se agrupó a los/as niños/as en tres categorías, los que obtuvieron: No satisfactorio (NS); Satisfactorio-Bueno (S-B); y. Muy Bueno-Excelente $(M B-E)$. Esta agrupación se utilizó para definir las trayectorias de rendimiento académico.

Trayectorias de rendimiento académico: refieren a las tendencias que siguen las calificaciones de los y las escolares a lo largo del periodo de tiempo estudiado. Para definirlas se tuvo en cuenta: a) la calificación del tercer trimestre en Lengua y Matemática (NS/B-S/MB-E) obtenida por los/as niños/as al inicio y al final del seguimiento, según hayan mejorado, mantenido o disminuido su RA de un momento a otro, y b) su condición final (Promovido/No promovido) en caso de que hayan rendido examen complementario. Se definieron cuatro tipos de trayectorias para cada asignatura: Distinguida: refiere a un desempeño del niño/a en la escuela que se ajusta a las exigencias académicas, obteniendo y manteniendo en el tiempo calificaciones sobresalientes en las instancias evaluativas observadas; incluye a los/as niños/as que, al inicio y al final del seguimiento, mantuvieron calificaciones $\mathrm{E}-\mathrm{MB}$, tuvieron E-MB pero disminuyeron a B-S, y aquellos que teniendo calificaciones B-S 
mejoraron a E-MB; Satisfactoria: se trata de un desempeño del niño/a suficiente para cumplir con los requerimientos de la escuela manteniendo las calificaciones medias de la escala de calificación; incluye los escolares que mantuvieron calificaciones B-S durante el periodo estudiado; Regular: se trata de una experiencia escolar que va al ritmo establecido por la escuela pero con dificultad, requiriendo instancias y esfuerzos complementarios para la obtención de calificaciones satisfactorias, por lo que roza el límite del riesgo educativo. Incluye a los/as niños/as que, a lo largo del periodo estudiado, tenían NS y mejoraron su rendimiento a B-S, los/as que mantuvieron calificaciones NS pero que aprobaron el examen complementario siendo promovidos/as de grado, y aquellos escolares con calificaciones satisfactorias que disminuyeron su rendimiento a NS pero que también aprobaron el examen complementario y promovieron de grado; Insuficiente: refiere una experiencia educativa signada por bajos rendimientos, y como consecuencia de ello, a niños/as en riesgo educativo; incluye a los/as niños/ as con calificaciones NS que desaprobaron el examen complementario y que, durante el periodo estudiado, repitieron de grado al menos una vez.

Escolaridad materna: fue informada por los adultos responsables de los/as niños/as mediante una encuesta elaborada a tal fin. La escolaridad materna se definió por el nivel escolar más alto completado por la madre de acuerdo con la Clasificación Internacional Normalizada de la Educación (UNESCO, 2012), a partir del cual se conformaron dos niveles de categorías: madres con educación secundaria incompleta o menos, y madres con educación secundaria completa o más.

Estrato ocupacional: la categoría ocupacional de la persona principalmente responsable del sustento familiar (sostén del hogar) fue informada por los adultos responsables de los/as niños/as, y se utilizó para definir estratos ocupacionales relacionados con la estratificación social de la Argentina (Sabulsky y otros, 1995): el estrato alto incluyó a las categorías ocupacionales de empleadores, profesionales y asalariados jerárquicos, el estrato medio a trabajadores por cuenta propia y asalariados estables cuyo trabajo no es 
manual; el estrato bajo a asalariados estables cuyo trabajo es manual, y el estrato muy bajo a asalariados no calificados y trabajadores temporarios.

Nivel socioeconómico (NSE): se indagó mediante la escala Family Affluence Scale (FAS) (Currie et al., 2008), que evalúa la disponibilidad en el hogar (y el número) de autos y computadoras, habitación propia e individual del niño/a y vacaciones familiares en los últimos 12 meses. Es ampliamente utilizada en encuestas autoadministradas a escolares por el alto porcentaje de respuestas de niños/as y adolescentes, así como por tener una aceptable correlación con la categoría ocupacional de los padres y el nivel de ingresos de la familia (Boyce, Torsheim, Currie y Zambon, 2006). El puntaje del FAS se categorizó en: NSE bajo (puntaje: 0-3), medio (puntaje: 4-5), y alto (puntaje: 6-9).

Capacidad intelectual (Cl): fue medida con el Test de Matrices Progresivas de Raven (Escala Coloreada) (Raven, Court y Raven, 1993), el cual se administró de manera colectiva y en aula, corroborándose previamente la consistencia interna, fiabilidad test-retest y validez de constructo del test aplicado de esta manera (Degoy y Berra, 2016). Esta prueba evalúa el razonamiento analógico, la percepción y la capacidad de abstracción de los niños/as participantes permitiendo obtener una medida general de inteligencia. Puntuaciones iguales o menores al percentil 50 (P50) del conjunto de esta muestra se consideró una capacidad intelectual inferior al término medio; P50 hasta P75, una capacidad intelectual de término medio; y puntuaciones iguales o mayores al P75, una capacidad intelectual superior al término medio.

\section{Análisis de datos}

Se realizó un análisis descriptivo de las características de la muestra en el momento basal y en el seguimiento calculando las distribuciones de frecuencias de las variables estudiadas. Las asociaciones entre variables se exploraron utilizando el test de chi cuadrado, con un nivel de significación estadística en el valor de $p<0.05$. Modelos de regresión logística binomial y multinomial con odds ratios (OR) e intervalos de confianza del 95\%, 
fueron utilizados para examinar la presencia de desigualdades sociales en las trayectorias de rendimiento académico, ajustando por capacidad intelectual. Los datos recopilados se analizaron utilizando la versión 18 de SPSS para Windows.

\section{Resultados}

En el momento inicial participaron 494 escolares y en el seguimiento 438 (tasa de respuesta: $82,2 \%$ ). La edad media de los/as niños/as al inicio del estudio fue de 9,5 años (Desviación Estándar $(D E)=0,65 ;$ Rango $=9$ a 12 años), y de 11,6 años al final del seguimiento ( $D E=0,66$; Rango= 11 a 14 años). El 15\% de los escolares presentó sobreedad, es decir una edad superior a la considerada teórica para el grado que cursan. Al inicio del estudio, hubo una proporción similar de niños y niñas, las madres del $65 \%$ de los escolares informaron un nivel de escolaridad de secundaria incompleta o menos, el $50 \%$ de las personas sostén de hogar se clasificaron en el estrato ocupacional muy bajo, y el $14 \%$ de los/as niños/as declaró bajos recursos económicos familiares ubicándose en el nivel socioeconómico inferior. Porcentajes similares se observaron entre los/as participantes del seguimiento a los 24 meses. En comparación con los/as no participantes, los/ as participantes del seguimiento mostraron una mayor capacidad intelectual y mejor rendimiento académico tanto en Lengua como en Matemática. No se encontraron diferencias estadísticamente significativas entre aquellos/as que participaron del seguimiento y quienes no, según sexo, edad escolar, escolaridad materna, estrato ocupacional y NSE (Tabla 1). 
Tabla 1.

Características de la muestra en momento inicial y según participación en el seguimiento a los 24 meses. Periodo 2014-2016.

\begin{tabular}{|c|c|c|c|c|c|c|c|}
\hline & \multicolumn{2}{|c|}{ Inicio } & \multicolumn{2}{|c|}{$\begin{array}{l}\text { Participantes del } \\
\text { seguimiento }\end{array}$} & \multicolumn{2}{|c|}{$\begin{array}{c}\text { No participantes del } \\
\text { seguimiento }\end{array}$} & \multirow[t]{2}{*}{$\mathbf{P}^{\text {a }}$} \\
\hline & $\%$ & (n) & $\%$ & (n) & $\%$ & (n) & \\
\hline \multicolumn{8}{|l|}{ Sexo } \\
\hline Masculino & 51 & (252) & 50 & (211) & 58 & (41) & \multirow{2}{*}{0.136} \\
\hline Femenino & 49 & (242) & 50 & $(212)$ & 42 & (30) & \\
\hline \multicolumn{8}{|l|}{ Edad escolar } \\
\hline Edad teórica & 85 & (412) & 86 & (362) & 78 & $(50)$ & \multirow{2}{*}{0.091} \\
\hline Sobreedad & 15 & $(75)$ & 14 & $(61)$ & 22 & (14) & \\
\hline \multicolumn{8}{|l|}{ Escolaridad materna } \\
\hline$\leq$ Sec. incompleto & 65 & (289) & 66 & (261) & 57 & (28) & \multirow{2}{*}{0.151} \\
\hline$\geq$ Sec. completo & 35 & (157) & 34 & (136) & 43 & (21) & \\
\hline \multicolumn{8}{|l|}{ Estrato ocupacional } \\
\hline Muy Bajo & 50 & (177) & 50 & (157) & 44 & (20) & \multirow{3}{*}{0.441} \\
\hline Bajo & 29 & (104) & 29 & $(92)$ & 27 & (12) & \\
\hline Medio & 21 & $(76)$ & 21 & (63) & 29 & (13) & \\
\hline Alto & 0 & $(0)$ & 0 & $(0)$ & 0 & $(0)$ & \\
\hline \multicolumn{8}{|l|}{ Nivel socioeconómico } \\
\hline Bajo & 14 & $(68)$ & 14 & $(58)$ & 14 & (10) & \multirow{3}{*}{0.284} \\
\hline Medio & 51 & (249) & 50 & (208) & 59 & (41) & \\
\hline Alto & 35 & (173) & 36 & (173) & 27 & (19) & \\
\hline \multicolumn{8}{|l|}{ Capacidad intelectual } \\
\hline Inferior & 21 & $(85)$ & 19 & $(72)$ & 46 & (13) & \multirow{3}{*}{0.002} \\
\hline Término medio & 54 & $(225)$ & 56 & $(215)$ & 36 & (10) & \\
\hline Superior & 25 & (104) & 26 & $(99)$ & 18 & $(5)$ & \\
\hline \multicolumn{8}{|l|}{ Rendimiento Lengua } \\
\hline E-MB & 30 & (148) & 31 & (133) & 21 & (15) & \multirow{3}{*}{$<0.001$} \\
\hline B-S & 60 & (294) & 61 & $(257)$ & 52 & (37) & \\
\hline NS & 10 & $(52)$ & 8 & (33) & 27 & (19) & \\
\hline \multicolumn{8}{|c|}{ Rendimiento Matemática } \\
\hline E-MB & 32 & (156) & 33 & $(138)$ & 25 & (18) & \multirow{3}{*}{$<0.001$} \\
\hline B-S & 58 & (289) & 61 & (258) & 44 & (31) & \\
\hline NS & 10 & $(49)$ & 6 & $(27)$ & 31 & $(22)$ & \\
\hline
\end{tabular}

${ }^{a}$ Valor $p$ prueba chi cuadrado

La proporción de escolares que fueron clasificados en el rango de una $\mathrm{Cl}$ superior al término medio fue similar entre niños (24\%) y niñas (25\%). Se confirmó asociación entre la capacidad intelectual y todas las características socioeconómicas analizadas. Aquellos niños/as cuyas madres reportaron un nivel de escolaridad de secundario incompleto o menos $(\mathrm{OR}=0,31$; $\mathrm{IC} 95 \%=0,19-0,49)$, cuyo sostén del hogar se ubica en el estrato ocupacional categorizado como muy bajo (OR=0,44; IC95\%=0,23-0,83), y que refirieron un menor NSE $(\mathrm{OR}=0,33$; IC95\%=0,14-0,78), presentaron menos posibilidades de un $\mathrm{Cl}$ superior al término medio que aquellos escolares con madres con mayores niveles de escolaridad, de estrato ocupacional medio, y NSE alto (Tabla 2). 
Tabla 2.

Asociación entre características socioeconómicas y capacidad intelectual en escolares de la ciudad de Córdoba-Argentina.

Periodo 2014-2016.

\begin{tabular}{|c|c|c|c|c|c|c|c|c|c|}
\hline & \multicolumn{6}{|c|}{ Capacidad Intelectual } & \multirow{3}{*}{$\mathrm{p}^{\mathrm{a}}$} & \multirow{2}{*}{\multicolumn{2}{|c|}{$\begin{array}{l}\mathrm{Cl} \text { Superior al término } \\
\text { medio vs resto }{ }^{\text {b }}\end{array}$}} \\
\hline & \multicolumn{2}{|c|}{ Superior } & \multicolumn{2}{|c|}{ Término medio } & \multicolumn{2}{|c|}{ Inferior } & & & \\
\hline & $\%$ & $(n)$ & $\%$ & (n) & $\%$ & $(n)$ & & OR & (IC 95\%) \\
\hline \multicolumn{9}{|l|}{ Sexo } & \multirow{3}{*}{$(0.63-1.52)$} \\
\hline Masculino & 24 & $(52)$ & 51 & $(108)$ & 25 & (54) & \multirow{2}{*}{0.044} & 0.98 & \\
\hline Femenino & 25 & (53) & 60 & (128) & 15 & (34) & & 1 & \\
\hline Total & 24 & (105) & 55 & (236) & 21 & (88) & & & \\
\hline \multicolumn{9}{|l|}{ Escolaridad materna } & \multirow{3}{*}{$(0.19-0.49)$} \\
\hline$\leq$ Sec. incompleto & 17 & (44) & 56 & $(147)$ & 27 & $(71)$ & \multirow{2}{*}{$<0.001$} & 0.31 & \\
\hline$\geq$ Sec. completo & 40 & (54) & 51 & (69) & 10 & (13) & & 1 & \\
\hline Total & 25 & $(98)$ & 54 & (216) & 21 & (84) & & & \\
\hline \multicolumn{9}{|l|}{ Estrato ocupacional } & \multirow[b]{2}{*}{$(0.23-0.83)$} \\
\hline Muy bajo & 22 & (33) & 56 & $(85)$ & 23 & (35) & & 0.44 & \\
\hline Bajo & 27 & (23) & 52 & (44) & 21 & (18) & 0.048 & 0.59 & $(0.29-1.18)$ \\
\hline Medio & 38 & (25) & 52 & (34) & 9 & (6) & & 1 & \\
\hline Total & 27 & $(81)$ & 54 & $(163)$ & 19 & (59) & & & \\
\hline \multicolumn{9}{|c|}{ Nivel socioeconómico (FAS) } & \multirow{3}{*}{$\frac{(0.14-0.78)}{(0.46-1.19)}$} \\
\hline Bajo & 13 & $(7)$ & 58 & $(32)$ & 29 & (16) & & 0.33 & \\
\hline Medio & 25 & $(51)$ & 53 & $(109)$ & 22 & $(46)$ & 0.047 & 0.74 & \\
\hline Alto & 31 & $(46)$ & 54 & $(81)$ & 15 & $(23)$ & & 1 & \\
\hline Total & 25 & $(104)$ & 54 & $(222)$ & 21 & (85) & & & \\
\hline
\end{tabular}

a Valor de p en prueba chi cuadrado

b Modelo de regresión logística binaria. Evento: $\mathrm{Cl}$ superior al término medio.

La Figura 1 presenta la distribución de frecuencias de las calificaciones del tercer trimestre en Lengua y Matemática, y su progresión durante los dos años de seguimiento señalando la proporción de niños/as que, de un ciclo lectivo a otro, mejoran, mantienen o disminuyen su rendimiento académico. Allí puede observarse que al inicio del estudio, un 32\% de los escolares presentó calificaciones E-MB en Lengua. La mayoría de ellos (69\%) mantuvo esta calificación de un año a otro, y los que disminuyeron su rendimiento (31\%) lo hicieron obteniendo calificaciones B-S, pero ninguno/a de ellos/ as llegó a desaprobar la asignatura. Respecto al grupo con NS en Lengua, se observa una disminución de la proporción de niños/as con estas calificaciones desde el momento basal a los 24 meses (que va de un $8 \%$ a $2 \%$ ), lo cual indica que este grupo mejora su rendimiento a lo largo del seguimiento, pero ninguno/a de ellos/as logra obtener las calificaciones máximas de E-MB. Tendencias similares a las descriptas se observan en Matemática, en ambos grupos de rendimiento. 


\section{Figura 1.}

Distribución de frecuencias de las calificaciones del tercer trimestre en Lengua y Matemática, y su progresión durante el periodo de seguimiento (2014-2016).

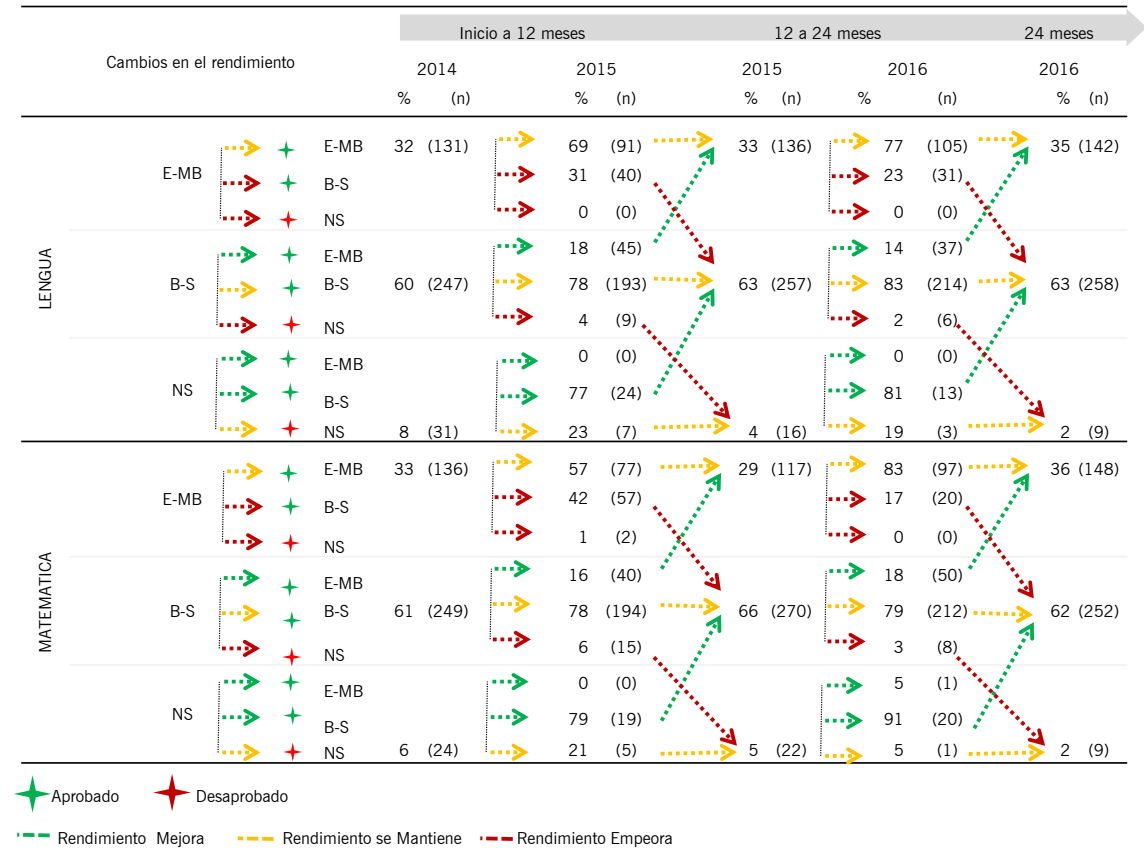

El 37\% $(n=166)$ de los escolares presentó una trayectoria distinguida, el $50 \%(n=226)$ satisfactoria, el $8 \%(n=36)$ regular y el $5 \%(n=26)$ insatisfactoria (Tabla 3). Se encontraron diferencias estadísticamente significativas $(p<0.05)$ en la trayectoria de rendimiento académico según sexo, escolaridad materna, NSE y capacidad intelectual. Las niñas presentaron un mejor rendimiento que los niños, ya que se observó una mayor proporción de niñas (44\%) que de niños (30\%) con trayectoria distinguida, y una mayor proporción de niños (11\%) que de niñas (5\%) con trayectoria regular. Por otro lado, la trayectoria distinguida se observó con mayor frecuencia entre aquellos/as niños/as cuyas madres alcanzaron una escolaridad de secundario completo o más que entre aquellos/as con madres con niveles de escolaridad 
más bajos (48\% vs 32\%), con padres/madres del estrato ocupacional medio en comparación con los de estrato ocupacional bajo o muy bajo (53\% vs $37 \%$ ), entre los/as niños/as que informaron un mejor NSE que entre los/ as que informaron un peor NSE ( $45 \%$ vs $24 \%$ ), y entre aquellos/as con puntuaciones más altas en el test de capacidad intelectual que entre los que obtuvieron puntuaciones más bajas ( $58 \%$ vs $15 \%$ ). Las trayectorias regulares e insatisfactorias de los escolares fueron más frecuentes entre los niños y niñas de niveles más bajos de escolaridad materna, estrato ocupacional, NSE y capacidad intelectual (Tabla 3 ).

\section{Tabla 3.}

Distribución de frecuencias de las trayectorias de rendimiento académico en función de característica socioeconómicas y capacidad intelectual en escolares de la ciudad de Córdoba, Argentina. Periodo 2014-2016.

\begin{tabular}{|c|c|c|c|c|c|c|c|c|c|c|}
\hline & \multirow[t]{2}{*}{$\mathrm{n}$} & \multicolumn{2}{|c|}{$\begin{array}{l}\text { Trayectoria } \\
\text { Distinguida }\end{array}$} & \multicolumn{2}{|c|}{$\begin{array}{l}\text { Trayectoria } \\
\text { Satisfactoria }\end{array}$} & \multicolumn{2}{|c|}{$\begin{array}{c}\text { Trayectoria } \\
\text { Regular }\end{array}$} & \multicolumn{2}{|c|}{$\begin{array}{c}\text { Trayectoria } \\
\text { Insatisfactoria }\end{array}$} & \multirow[t]{2}{*}{$P^{a}$} \\
\hline & & $\%$ & (n) & $\%$ & (n) & $\%$ & (n) & $\%$ & $(n)$ & \\
\hline \multicolumn{11}{|l|}{ Sexo } \\
\hline Masculino & 223 & 30 & (66) & 54 & (120) & 11 & (25) & 5 & (12) & \multirow{3}{*}{0.004} \\
\hline Femenino & 229 & 44 & (100) & 46 & (106) & 5 & (11) & 5 & (12) & \\
\hline Total & 452 & 37 & (166) & 50 & $(226)$ & 8 & (36) & 5 & $(24)$ & \\
\hline \multicolumn{11}{|l|}{ Escolaridad materna } \\
\hline$\leq$ Sec. incompleto & 284 & 32 & (91) & 53 & (149) & 9 & (23) & 7 & (19) & \multirow{3}{*}{0.005} \\
\hline$\geq$ Sec. completo & 141 & 48 & (68) & 43 & (61) & 6 & (9) & 2 & (3) & \\
\hline Total & 425 & 37 & (159) & 49 & (210) & 8 & (34) & 5 & (22) & \\
\hline \multicolumn{11}{|l|}{ Estrato ocupacional } \\
\hline Muy Bajo & 160 & 37 & (59) & 48 & (76) & 11 & (17) & 5 & (8) & \\
\hline Bajo & 94 & 37 & (35) & 53 & $(50)$ & 7 & $(7)$ & 2 & (2) & \multirow{3}{*}{0.135} \\
\hline Medio & 64 & 53 & (34) & 42 & $(27)$ & 3 & $(2)$ & 2 & (1) & \\
\hline Total & 318 & 40 & (128) & 48 & (153) & 8 & (26) & 3 & $(11)$ & \\
\hline \multicolumn{11}{|c|}{ Nivel socioeconómico (FAS) } \\
\hline Bajo & 59 & 24 & (14) & 59 & (35) & 7 & (4) & 10 & (6) & \\
\hline Medio & 215 & 34 & (73) & 54 & $(116)$ & 8 & (18) & 4 & (8) & \multirow{3}{*}{0.041} \\
\hline Alto & 159 & 45 & $(71)$ & 43 & (69) & 8 & $(12)$ & 4 & $(7)$ & \\
\hline Total & 433 & 37 & (158) & 52 & (220) & 8 & (34) & 5 & $(21)$ & \\
\hline \multicolumn{11}{|l|}{ Capacidad intelectual } \\
\hline Inferior & 79 & 15 & (12) & 63 & (50) & 11 & (9) & 10 & (8) & \\
\hline Término medio & 225 & 39 & $(87)$ & 50 & $(112)$ & 7 & (16) & 4 & $(10)$ & \multirow{2}{*}{$-b$} \\
\hline Superior & 101 & 58 & (59) & 39 & (39) & 3 & (3) & 0 & (0) & \\
\hline Total & 405 & 39 & (158) & 50 & (201) & 7 & $(28)$ & 4 & (18) & \\
\hline
\end{tabular}

${ }^{a}$ Valor $\mathrm{p}$ prueba chi cuadrado

${ }^{\text {b }}$ Se omite la prueba chi cuadrado porque la frecuencia de una de las casillas en menor a 5 
Finalmente, en la Tabla 4, se presenta el análisis multivariado y multinomial, que permite observar la asociación de las principales variables del estudio, donde se destaca la fuerte asociación entre las variables socioeconómicas y las trayectorias regular e insatisfactoria, teniendo a la trayectoria distinguida como referencia. Las trayectorias regular e insatisfactoria, tal y como fueron aquí definidas, remiten a niños y niñas en riesgo educativo (UNESCO-UNICEF, 2012). La trayectoria regular hace referencia a escolares con bajo rendimiento académico, y por lo tanto, con dificultades en la calidad de los aprendizajes adquiridos, mientras que la trayectoria insatisfactoria remite a escolares con dificultades tanto en la calidad como en los ritmos de aprendizaje, ya que incluyó a quienes repitieron de grado.

Puede observarse asociación entre el sexo masculino y el estrato ocupacional muy bajo con la trayectoria de rendimiento académico regular. Los niños, en comparación con las niñas ( $O R=3,44$; IC95\%=1,58-7,46), y los escolares con padres/madres con un estrato ocupacional muy bajo, en comparación con los de estrato ocupacional medio ( $O R=4,89$; IC95\% $=1,06-22,50)$, presentaron más chances de una trayectoria regular que una trayectoria distinguida. Por otro lado, niveles de escolaridad materna y NSE más bajos se encontraron fuertemente asociados a una trayectoria insatisfactoria del rendimiento escolar. Aquellos niños/as con madres con una escolaridad de secundario incompleto o menos, en comparación con los/as que tienen mamás con escolaridad de secundario completo o más, tienen una probabilidad 4,73 veces superior de presentar una trayectoria de RA insatisfactoria antes que una trayectoria distinguida. En la misma dirección, al comparar los escolares de un NSE bajo con los de un NSE alto, los/as niños/as de menores recursos presentan 4,34 veces más chances de presentar una trayectoria insatisfactoria que una trayectoria de RA distinguida. En cuanto a la capacidad intelectual, se observó que a medida que aumenta el puntaje obtenido en la prueba de $\mathrm{Cl}$, las posibilidades de obtener una trayectoria satisfactoria (OR=0,86; IC95\%=0,82-0,91), r egular ( $O R=0,83 ;$ IC95\%=0,77-0,90) o insatisfactoria $\mathrm{OR}=0,75$; IC95\% $=0,69-0,82$ ) son menores que la de obtener una trayectoria distinguida. Sin embargo, al ajustar los modelos por capacidad intelectual, se observa que las $\mathrm{OR}$ de las variables sociales se mantienen altas aunque pierden significación estadística. 


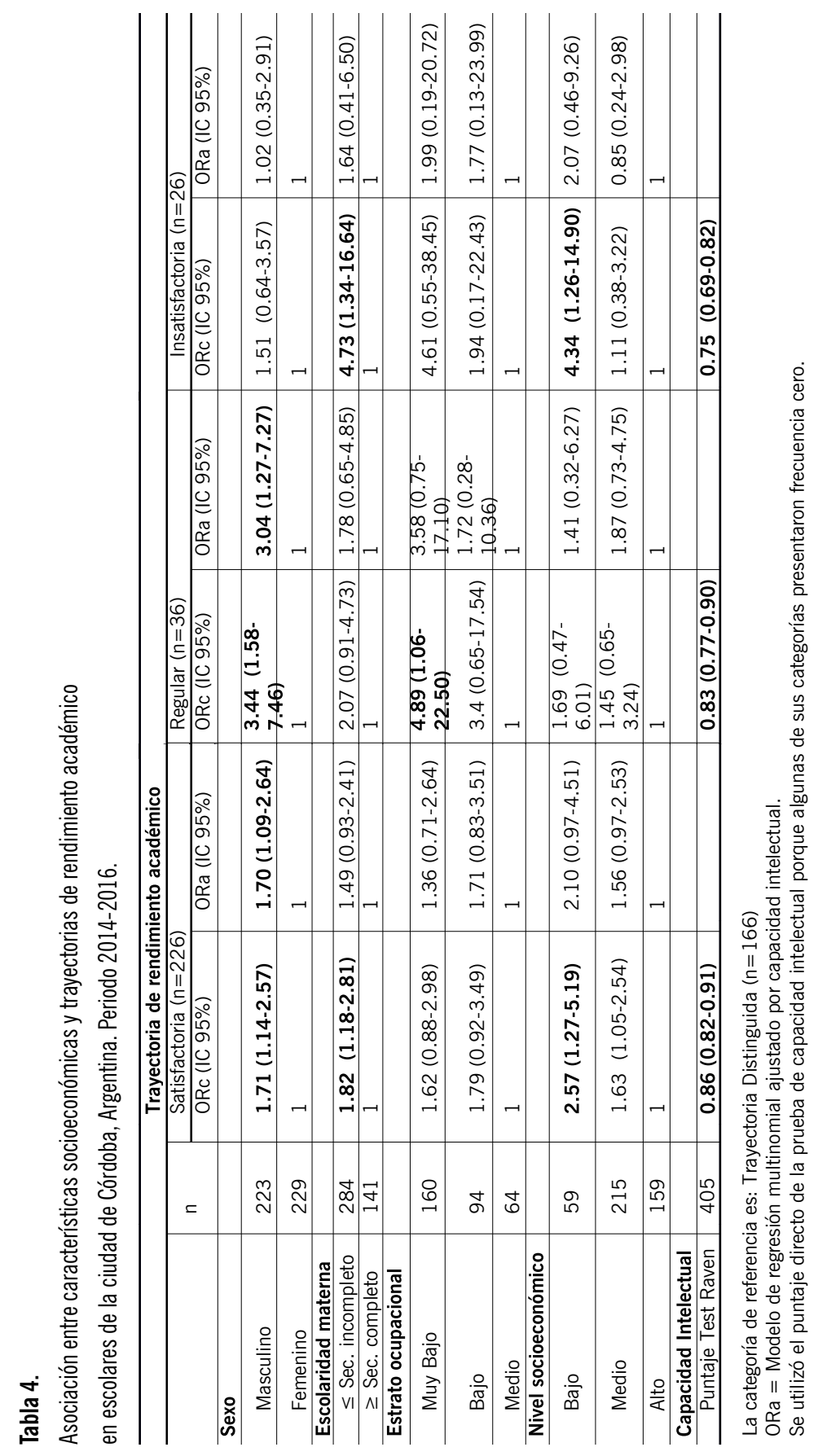




\section{Conclusiones}

Los resultados de este estudio dan cuenta de la presencia de desigualdades sociales en las trayectorias de rendimiento académico de niños/as asistentes a escuelas primarias públicas de la ciudad de Córdoba-Argentina. Se observó que las variables socioeconómicas aquí consideradas tienen impacto sobre las trayectorias de rendimiento académico, pero que su efecto no es independiente de la capacidad intelectual. A su vez, la capacidad intelectual de los escolares se encontró fuertemente asociada con la escolaridad materna, el estrato ocupacional y el nivel socioeconómico de las familias, viéndose desfavorecidos en las posibilidades de desarrollo de esta capacidad los niños y niñas de sectores más vulnerables. Esto sugiere que hay una desigualdad anterior $\mathrm{a}$, y fundante de, las que se dan dentro de la escuela, y que tienen que ver con desigualdades sociales en las posibilidades de desarrollo de las capacidades cognitivas necesarias para poder cumplir satisfactoriamente con las exigencias de la escuela y trasformar las oportunidades educativas en resultados.

La trayectoria regular fue más frecuente en niños. Las diferencias en el rendimiento académico entre varones y mujeres han sido reportadas en estudios previos (Echavarri, Godoy y Olaz 2007; Myhill y Jones, 2006) que, al igual que el presente trabajo, coinciden en señalar un mejor rendimiento académico de las mujeres. La hipótesis de la semejanza de género propuesta por Hyde (2005) sugiere que niños y niñas son más parecidos que diferentes en la mayoría de las variables cognitivas (Zell, Krizan y Teeter, 2015; Hyde, 2014). Los resultados de este estudio son congruentes con esta hipótesis, ya que no se encontraron diferencias por sexo en la capacidad intelectual de los escolares, siendo las diferencias en las trayectorias de rendimiento académico entre niños y niñas significativas independientemente de la $\mathrm{Cl}$. Existen estudios que han posicionado el debate sobre el bajo rendimiento de los niños como una cuestión de género, y lo describen como una respuesta a la cultura masculina en la que se encuentran inmersos (Younger, Warrington y Williams, 1999). El escenario escolar aparece aquí como un espacio de demostración de masculinidad, en el que los varones manifestarían un sí mismo masculinizado de diversas maneras, como por ejemplo, a través del desafío a las figuras de autoridad, la confrontación abierta con los docentes, 
por medio de la desestimación de los asuntos académicos, rudeza e impulsividad en los tratos; comportamientos que son legitimados y tolerados en tanto ejercidos por niños y considerados masculinos, pero que van en contra de lo que la escuela suele calificar como propio de un buen alumno. A su vez, las maestras también se encuentran atravesadas por las construcciones de género y la idea de que niños y niñas son diferentes en cuanto a actitudes hacia la escuela, motivación, madurez, responsabilidad, comportamiento e identificación con el espíritu escolar. Los niños son vistos como más negativos y necesitando competencia, disciplina, estructura y apoyo; las niñas, como más trabajadoras, teniendo una mejor motivación, siendo más cooperativas en el aula y estando mejor organizadas con respecto a la tarea (Arnot y Gubb, 2001, en Myhill y Jones, 2006). Así, el niño inteligente pero inquieto, disruptivo, y de bajo rendimiento, y la niña estudiosa, tranquila, obediente, y de alto rendimiento, se describen conforme a las normas de género y son considerados como típicos de su género. Según señalan Jones y Myhill (2004), estas construcciones de identidad de género producen expectativas estereotipadas y diferenciales sobre el comportamiento y los logros académicos de niños y niñas, que influyen en el trato que se le da en el aula y en la evaluación que se hace de ellos y ellas, situando a los niños como más propensos a ser elegidos como estudiantes que no alcanzan resultados escolares esperados.

La variable de estrato ocupacional considerada en este estudio, que se asoció con la trayectoria regular, responde a la clase social, esto es, al sistema de estratificación que clasifica a las personas por su acceso diferencial a recursos materiales, sociales y culturales. La investigación sobre estereotipos asociados a la clase social ha sido poco desarrollada hasta ahora, a pesar de ser un determinante fundamental del curso de la vida de cualquier individuo. Algunos estudios (Durante y Fiske, 2017; Croizet y Millet, 2012; Gorski, 2012) sostienen que, en diversos contextos, las personas de estratos sociales más bajos son consideradas muchas veces como menos competentes, vagas y poco interesadas por la educación que las de estrato alto, y que estos estereotipos impactan en el desempeño de distintas pruebas de rendimiento y capacidad. Se ha documentado también, que las personas de estratos sociales más bajos expuestas a estos estereotipos de clase 
informan una mayor ansiedad ante los exámenes, una menor confianza en sus capacidades y una menor identificación con los dominios académicos (Spencer y Castano, 2007), lo que podría explicar que los y las escolares con padres de estrato ocupacional más bajo presenten resultados académicos inferiores respecto de aquellos/as de estrato más alto.

La trayectoria insatisfactoria se observó con mayor frecuencia en escolares de bajos recursos socioeconómicos y con madres con menores niveles de escolaridad. Hertzman y Power (2003) sostienen que las circunstancias socioeconómicas y el capital cultural de las familias afectan a los/as niños/as porque dan forma a sus entornos próximos y a sus interacciones con ellos, ya que condicionan el acceso a espacios, tanto físicos como sociales, que les provean la estimulación, apoyo y cuidados necesarios para el máximo desarrollo de sus capacidades. Por su parte, Taylor, Clayton y Rowley (2004) han propuesto el concepto de socialización académica proporcionando un marco para comprender cómo la situación socioeconómica, y en particular la escolarización de las madres, influyen en los logros académicos de sus hijos/as. La socialización académica refiere al conjunto de procesos mediante los cuales, dentro del núcleo familiar, se moldean comportamientos, creencias, habilidades y actitudes de los/as niños/as para promover experiencias escolares exitosas. Así, por ejemplo, se ha observado que madres con mayores niveles de educación crean ambientes enriquecedores de lenguaje y alfabetización exponiendo a los/as niños/as a un lenguaje y vocabulario complejo, involucrando a los/as niños/as en actividades de aprendizaje tales como lectura, buscando oportunidades de aprendizaje estructurado, fomentando el pensamiento independiente y la resolución de problemas (Davis-Kean, 2005). En este sentido, una mejor situación socioeconómica y un mayor nivel de educación de los padres se traduce en un entorno más rico en recursos materiales y simbólicos que impactan en el desarrollo de las habilidades cognitivas y el desempeño escolar de niños y niñas. Las mejoras en la socialización académica que pueden resultar, particularmente del aumento de la educación materna, pueden ser especialmente importantes para los niños y niñas que se enfrentan a múltiples fuentes de desventaja y que ya comienzan la escuela en una situación de desventaja respecto de sus pares más favorecidos (Magnuson, 2007). 
De este modo, resulta interesante una lectura del rendimiento académico desde la teoría ecológica del desarrollo de Bronfenbrenner (1986), la cual postula que, aunque la familia es el contexto principal en el que tiene lugar el desarrollo humano, no deja de ser uno de los varios entornos en los que el desarrollo ocurre, y por lo tanto, sus procesos no son independientes de los que operan en los demás espacios. A la vez, reconoce que hay entornos externos a los/as niños/as, en los que ellos/as no pasan su tiempo, pero en los que ocurren hechos que afectan sus entornos inmediatos de desarrollo, como por ejemplo el mundo laboral y las redes de apoyo sociales de sus padres; y un macrosistema, el cual refiere al sistema de creencias y la organización política, social y económica de una determinada región, que configura la forma y modos de relación de los sistemas de menor orden anteriormente mencionados, condicionando también las posibilidades de desarrollo humano. La lectura del desempeño escolar desde esta perspectiva, permite considerar los procesos sociales proximales y distales que afectan la forma en que los niños y niñas se desempeñan en la escuela.

Los datos aquí aportados se circunscriben al ámbito de las escuelas públicas municipales de la ciudad de Córdoba, ya que la muestra no incluyó a niños/ as asistentes a escuelas públicas provinciales 0 a instituciones privadas. No obstante, dan cuenta de la magnitud de desigualdades sociales dentro de un sector que ya cuenta con cierta segregación, en tanto la población que asiste a las escuelas municipales presenta una situación de mayor desventaja socioeconómica respecto de los/as que concurren al resto de las escuelas de la ciudad. En este sentido, si la muestra fuera representativa del conjunto de escolares de Córdoba, incluyendo a los estratos sociales más altos y respondiendo a la perspectiva teórico metodológica que sustenta el presente estudio, la magnitud de las diferencias encontradas en la capacidad intelectual y las trayectorias de rendimiento académico sería mayor. Por otro lado, el tamaño de la muestra limita la precisión de las mediciones y las pruebas estadísticas, lo que puede sesgar lo observado respecto de la asociación entre las trayectorias de rendimiento académico y las variables sociales analizadas.

Gran parte de los abordajes del rendimiento académico han dejado en segundo plano las variables de índole social por considerarlas distales y difí- 
ciles de modificar (Caso-Niebla y Hernandez-Guzman, 2007). Sin embargo, una posible y necesaria ruta de intervención debe dirigirse a mejorar los recursos socioeconómicos y culturales de las familias (Magnuson, 2007). Mientras la tarea de la justicia educativa es lograr que las características socioeconómicas de los/as estudiantes y las características diferenciadas entre las escuelas no sean impedimento para que los niños y niñas tengan una educación que les provea mejores oportunidades de vida, la equidad como inclusión, implica la necesidad de generar en todos las capacidades mínimas necesarias para funcionar en la sociedad (López, 2005). Esto excede las responsabilidades individuales de alumnos/as, familias y escuelas, y atañe a una política integral de Estado. La reducción de las desigualdades en las condiciones socioeconómicas de las familias es una vía alternativa y necesaria de intervención de las políticas estatales para mejorar los resultados académicos de los más chicos, ya que podrían tener impacto en los entornos físicos y sociales en los que los/as niños/as son criados y, con ello, en las posibilidades del desarrollo de sus recursos cognitivos, determinantes de sus resultados académicos y de desigualdades sociales de la vida adulta. Los resultados que aquí se muestran aportan datos para promover iniciativas públicas destinadas a reducir el riesgo educativo. Se requiere de un análisis específico y situado para comprender los obstáculos que en la actualidad presenta el acceso al derecho social a la educación, y avanzar en futuras investigaciones y políticas públicas que aborden la problemática del desempeño escolar desde una perspectiva más amplia e integral, como por ejemplo la que ofrece la teoría ecológica. Tener en cuenta las influencias externas que afectan la capacidad de familias y escuelas para fomentar el desarrollo pleno de los más pequeños, permitiría alternativas de intervención superadoras de las que, tradicionalmente, se han dirigido a intervenir directamente con niños/as en riesgo de fracaso escolar mediante acciones predominantemente paliatorias y con abordajes de tipo clínico e individual. 


\section{Referencias bibliográficas}

Boyce, W., Torsheim, T., Currie, C., y Zambon, A. (2006). The family affluence scale as a measure of national wealth: Validation of an adolescent self-report measure. Social Indicators Research, 78(3), 473-487. Briscioli, B. (2017). Aportes para la construcción conceptual de las «trayectorias escolares». Actualidades Investigativas en Educación, 17(3), 609-639.

Bronfenbrenner, U. (1986). Ecology of the family as a context for human development: Research perspectives. Developmental Psychology, (22) 723-742.

Cardini, A., Sánchez, B. y Rivas, A. (2016). Reflexiones sobre Aprender 2016. Buenos Aires: CIPPEC. Disponible en: http://www.cippec.org/publicacion/reflexiones-sobre-aprender/.

Caso-Niebla, J., y Hernández, L. (2007). Variables que inciden en el rendimiento académico de adolescentes mexicanos. Revista latinoamericana de psicología, 39(3), 487-501.

Cervini, R (2002). Desigualdades en el logro académico y reproducción cultural en Argentina. Revista Mexicana de Investigación Educativa, 7(16), 445-500.

Currie, C., Molcho, M., Boyce, W., Holstein, B., Torsheim, T., y Richter, M. (2008). Researching health inequalities in adolescents: the development of the Health Behaviour in School-Aged Children (HBSC) family affluence scale. Social Science y Medicine, 66(6), 1429-1436.

Croizet, J.C. y Millet, M. (2012). Social class and test performance: From stereotype threat to symbolic violence and vice versa. In M. Inzlicht y T. Schmader (Eds.), Stereotype threat: Theory, process, and application (pp. 188 -201). New York, NY: Oxford University Press. Davis-Kean, P. E. (2005). The influence of parent education and household income on child achievement: The indirect role of parental expectations and the home environment. Journal of Family Psychology, 19(2), 294304.
Degoy, E., \& Berra, S. (2018). Differences in health-related quality of life by academic performance in children of the city of Cordoba-Argentina. Quality of Life Research, 27(6), 1463-1471.

Degoy, E. y Berra, S. (2016). Fiabilidad y validez de la aplicación colectiva de un test de capacidad intelectual en escolares de la ciudad de Córdoba-Argentina. Presentación en el 18 Encuentro Nacional de Investigación Pediátrica de la Sociedad Argentina de Pediatría. Disponible en: http://www.sap.org.ar/docs/ congresos/2016/Investigaci\%C3\%B3n/programa_final_investigacion.pdf.

Durante, F. y Fiske, S. T. (2017). How social-class stereotypes maintain inequality. Current Opinion in Psychology, (18) 43-48.

Echavarri, M., Godoy, C. y Olaz, F. (2007). Diferencias de género en habilidades cognitivas y rendimiento académico en estudiantes universitarios. Universitas Psychologica, 6(2), 319-329.

Elder, G. (1994). Time, human agency, and social change: Perspectives on the life course. Social Psychology Quarterly, 57(1), 4-15.

Gertel, H., Giuliodori, R., Herrero, V., Fresoli, D., Vera, M.L. y Morra, G. (2006). Análisis multinivel del rendimiento escolar al término de la educación básica en Argentina. Anales de la XLI Reunión de la AAEP. www. aaep.org.ar.

González-Pienda, J. A. (2003). El rendimiento escolar. Un análisis de las variables que lo condicionan. Revista Galego-Portuguesa de Psicoloxía e Educación, (7) 240-247.

Gorski, P.C. (2012). Perceiving the Problem of Poverty and Schooling: Deconstructing the Class Stereotypes that Mis-Shape Education Practice and Policy. Equity y Excellence in Education, 45(2), 302-319. 
Greg, D., Morris, P. y Rodrigues, C. (2011). Does Money Really Matter? Esti mating Impacts of Family Income on Young Children's Achievement with Data from Random-Assignment Experiments. Developmental Psychology, 47(5), 1263-1279.

Hertzman, C., y Power, C. (2003). Health and human development: understandings from life-course research. Developmental Neuropsychology, 24(2-3), 719-44.

Hyde, J. S. (2005). The gender similarities hypothesis. American Psychologist, (60), 581-592.

Hyde, J. S. (2014). Gender similarities and differences. Annual Review of Psychology, (65), 373-398.

Jones, S. y Myhill, D. (2004). "Troublesome boys» and «compliant girls»: gender identity and perceptions of achievement and underachievement. British Journal of Sociology of Education, 25(5), 547-561.

Kuh, D., Ben-Shlomo, Y., Lynch J., Hallqvist J., Power, C. (2003). Life course epidemiology. Journal of Epidemiology Community Health, 57(10): 778-783.

Krüger, N. (2013). Segregación social y desigualdad de logros educativos en Argentina. Archivos Analíticos de Políticas Educativas, 21(86).

Lemos, G., Almeida, L.S., Guisande, M.A. y Primi, R. (2008). Intelligence and academic achievement: analysis of its relationship during schooling. Revista Portuguesa de Educação, 21(1), 83-99.

López, N. (2005). Equidad educativa y desigualdad social. Desafíos de la educación en el nuevo escenario latinoamericano. IIPE_ UNESCO, Bs As. Disponible en: http://unesdoc.unesco.org/images/0014/001425/142599s.pdf

Lynch, J.L., von Hippel, P.T. (2016). An education gradient in health, a health gradient in education, or a confounded gradient in both?. Social Science \& Medicine, (154) 18-27.

Magnuson, K. (2007). Maternal education and Children'snacademic achievement during middle childhood. Developmental Psychology, 43(6), 1497-1512.
Ministerio de Educación, Ciencia y Tecnología. (2005). Núcleos de aprendizajes prioritarios, 2do Ciclo EGB/ nivel primario. Disponible en: http://www.me.gov.ar/ curri form/publi ca/nap/nap_egb2.pdf.

Ministerio de Educación, Ciencia y Tecnología. (2016). Aprender: dispositivo nacional de evaluación de los aprendizajes de los estudiantes y de sistematización de información acerca de algunas condiciones en las que ellos se desarrollan. Disponible en: https://www. argentina.gob.ar/educacion/aprender.

Myhill, D. y Jones, S. (2006). "She doesn't shout at no girls': pupils' perceptions of gender equity in the classroom. Cambridge Journal of Education, 36(1), 99-113.

Navarro, R. (2003). El rendimiento académico: concepto, investigación y desarrollo. Revista Iberoamericana sobre Calidad, Eficacia y Cambio en Educación, 1(2). Noble, K., Norman, F., y Farah, M. (2005). Neurocognitive correlates of socioeconomic status in kindergarten children. Developmental Science, 8(1), 74-87.

Rajmil, L., Díez, E., Peiró, R. (2010). Desigualdades sociales en la salud infantil. Informe SESPAS 2010. Gaceta Sanitaria, (24), 42-48.

Raven, J. C., Court J. y Raven J. (1993). Test de matrices progresivas. Escalas coloreada. Manual. Bs. As.: Paidós.

Sabulsky, J., Batrouni, L., Carballo, R., Reyna, S., Quiroga, D., Roitter, H. D.,Gorostiaga, H. y Brizuela, M. (1995). Alimentación en el primer mes de vida por estratos sociales, Córdoba, Argentina. Boletín Oficina Sanitaria Panamericana, 119 (1), 15-27.

Sistema de Educación de Tendencias Educativas en América Latina - SITEAL (2017). Sistema de Educación de Tendencias Educativas en América Latina. Perfiles de País ARGENTINA. Disponible en: http://www. publicaciones.siteal.iipe.unesco.org/perfiles-de-pais/4/ argentina. 
Spencer , B. y Castano, E. (2007). Social class is dead. Long live social class! Stereotype threat among low socioeconomic status individuals. Social Justice Research, (20), 418-432.

Suhrcke, M., De Paz Nieves, C. (2011). The impact of health and health behaviors on educational outcomes in high-income countries: a review of the evidence. Copenhagen, Denmark: World Health Organization, Regional Office for Europe. 2011.

Terigi, F. (2009). El fracaso escolar desde la perspectiva psicoeducativa: hacia una reconceptualización situacional. Revista Iberoamericana de Educación, (50), 23-39.

Taylor, L.C., Clayton, J.D. y Rowley, S.J. (2004). Academic socialization: Understanding parental influences on children's school-related development in the early years. Review of General Psychology, 8(3), 163-178. UNESCO (2012). International Standard Classification of Education ISCED 2011. Montreal, Canada: UNESCO Institute for Statistics.

UNESCO-UNICEF (2012). Completar la escuela: un derecho para crecer, un deber para compartir. Recuperado de www.unesco.org/new/fileadmin/MULTIMEDIA/FIELD/Santiago/pdf/Informe-UNESCO-UNICEF-AGO-2012.pdf.

Younger, M., Warrington, M. y Williams, J. (1999). The gender gap and Classroom Interaction: reality and rhetoric? British Journal of Sociology of Education, 20(3), 325-341.

Zell, E., Krizan, Z. y Teeter, S.R. (2015). Evaluating gender similarities and differences using metasynthesis. American Psychologist, (70), 10-20. 